\title{
The Usage of the Standards into the Long Term Archiving and Retrieval, and the Exchange of Engineering Design Data
}

\author{
Frédéric Chambolle \\ PSA Peugeot Citroën, France \\ frederic.chambolle@mpsa.com
}

\begin{abstract}
Standards have been developed to provide means of exchange and archival of engineering design data within automotive industry. In most cases, the simple use of these standards is not enough. Indeed, the usage of such standards requires the development of recommendations and methodologies providing specific rules and processes that will guide the user on how the standard is to be used in a given context. This paper will present examples of data exchange problems with some examples of methodologies developed to solve them, and - automotive recommendations developed in several domains such as PDM data exchange, Product Data Quality, Long Term Arching and Retrieval, etc. how they are used within the industrial processes.
\end{abstract}

Keywords: Archival, Exchange, Standards, Automotive, STEP, OAIS, CAD, PLM, SASIG.

\section{Introduction}

The engineering development of complex products such as automotive products is made in an extended enterprise context with suppliers, but also with other car manufacturers in case of partnership projects related to the development of cars, engines, gear boxes, etc. Extended enterprise requires collaboration within a heterogeneous environment involving different actors using complex IT environments, implementing different business processes, etc.

In this context, data interoperability is a major issue. Data exchange standards are then key elements since they provide a software independent exchange solution.

Nevertheless, due to the complexity of their definition and implementation, the single usage of such standards cannot systematically lead to a successful data exchange. Software editors and automotive companies have decided to define recommendations in order to improve the technical and functional quality of data exchange. These recommendations mainly concern the domains of Computer Aided Design, Product Data Management, and Long Term Arching and Retrieval. They provide explicit and shared rules of implementation to allow a better use data exchange standards, or define processes to be applied upstream and downstream of the data exchange. 
Then, these recommendations are intended to be used and combined with the data exchange standard for a better control of the data exchange processes.

\section{The Data Exchange Standards}

A data exchange standard provides a means to exchange information between different IT software, in a given application context (ex : Computer Aided Design, Digital Mockup, etc.). It proposes a content that can be interpreted/decoded by any software of the concerned domain through a translation function that enables to establish a data mapping between the standard and their internal data model. A data exchange standard is also known as "neutral format".

In the engineering domain, different types of standards are used :

- Official standard: it is published by a standardization organization (AFNOR, ANSI, ISO),

- Defacto standard: it is defined by a "commercial" organization, but considered as a reference in its domain. Thus, it is used by wide population of actors of this domain,

- Recommendation: it is defined and published by a community of interest (SASIG, LOTAR, ...). It usually defines either explicit business use cases, or mechanisms, algorithms, etc. that are not yet covered by a standard. A recommendation may become the foundation of a future standard.

Usually, for reasons of strategy and efficiency, neutral formats are opposed to native formats. The usage of native formats for data exchange has an evident interest if both partners use the same software. If not, the data exchange based on native formats requires the usage of direct translators that translate a data in an original format into the target format. A famous comparison between direct and neutral translators is presented in Fig. 1.
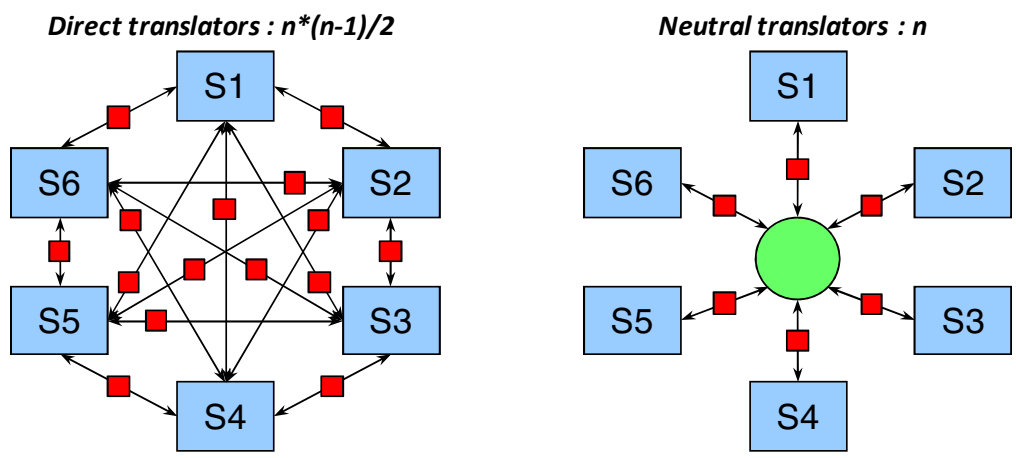

Fig. 1. Comparison amongst translators to implement in case of a direct or neutral translation strategy 
The most important figure concerns the number of translators, $n$ versus $n *(n-1) / 2$, that must be implemented to enable exchange between each software.

The usage of a direct translation constraints the user to upgrade its translators every time both original and target formats evolve (see Fig. 2). This has an impact on IT maintenance costs, and the stability of the translation processes.

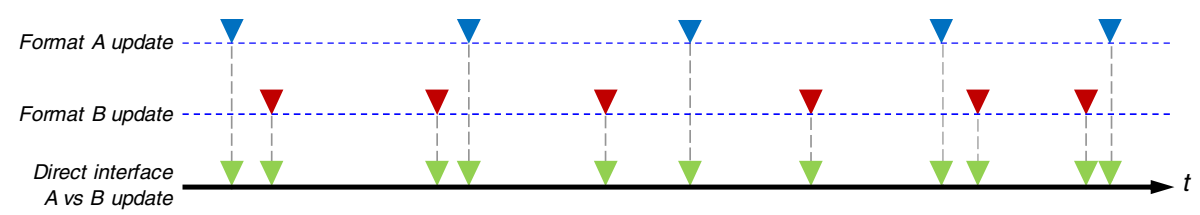

Fig. 2. Frequency of upgrades for direct translators

\section{CAD Data Exchange}

CAD domain is the most experienced domain in terms of data exchange. Indeed, many standards were developed since 70's in this domain (see Fig. 3).

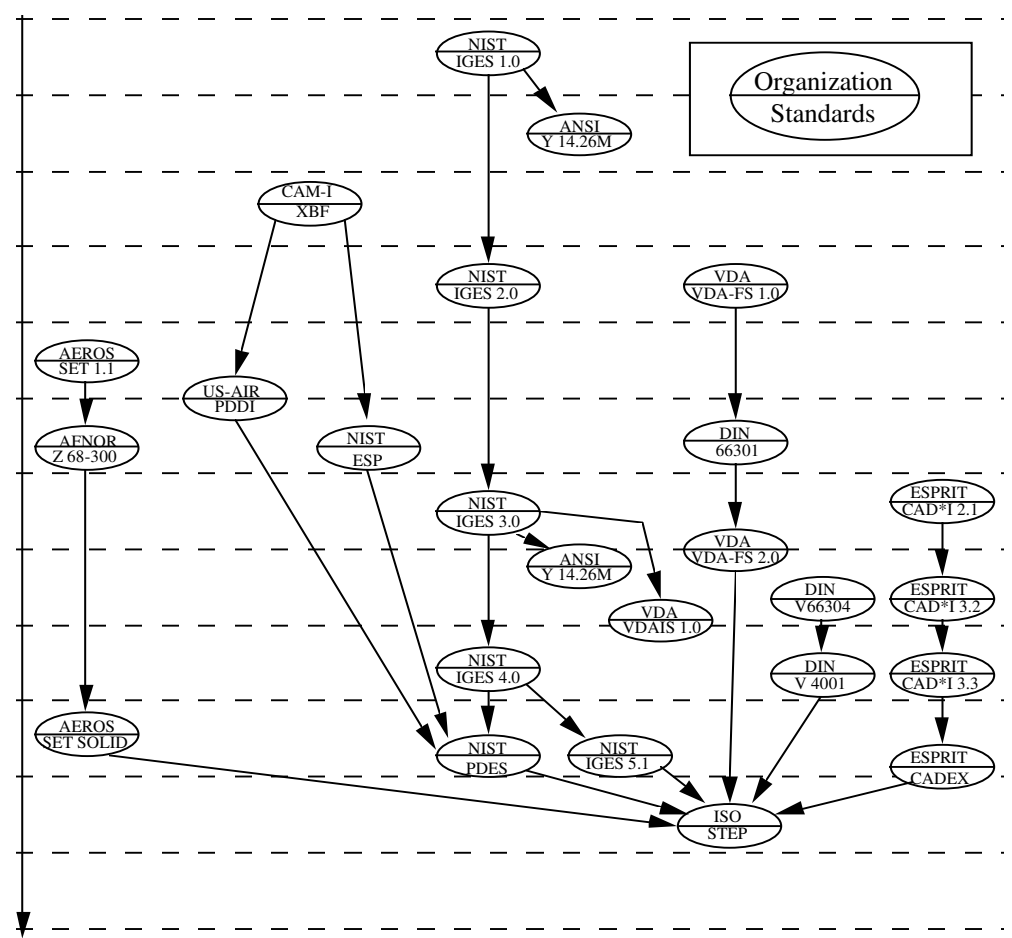

Fig. 3. History of CAD standards 
The richness of the content of the standard regularly increases. This evolution is driven by the CAD functionalities increasingly advanced (Construction history, parametric design, feature based modeling, etc.).

CAD translators have now reached a good level of quality. Several reasons enabled to reach that level of maturity :

- Lessons learnt from past experiences,

- Better standards,

- Implementor Forums.

For the development of STEP related translators, the CAx Implementor Forum [10] was created in 1995 with the support of PDES Inc. and ProSTEP iViP. The objective of the forum is to accelerate CAx translator development and ensure that users' requirements are satisfied. Thus, this forum enables software editors to :

- Organize cross testing sessions (CAD Round Table),

- Synchronize functionality implementation with the user needs,

- Identify the functionalities expected for "tomorrow",

- Establish agreement through the development of Recommended Practices : define clear use cases of the STEP standards (ex : Geometric and Assembly Validation Properties, Model styling and Organization, ...).

Nevertheless, even if CAD translators have reached a significant level of quality, data exchange rules needs to be implemented. This is the case in the automotive industry where each car manufacturer has developed its own CAD Data Exchange policy to be used into the data exchange processes with its suppliers. Such a policy defines CAD design rules and methodologies specific to a company, like file formats to be used, CAD model organization rules, accuracy, allowed types of entity, etc. These rules aim to optimize the integration of CAD design with processes downstream and upstream (meshing, release process, ...). The CAD Data Exchange policy is a contractual document that applies in the OEM-suppliers relationship.

Most of the rules contained in this document are implemented in CAD data quality checker that is used to verify whether the CAD design rules are satisfied at any stage of the design process. This process is mainly implemented to avoid any kind of problem in the downstream processes due to a poor quality of CAD data [5].

The international automotive industry, through the international consortium SASIG [9], has decided in 2000, to develop a recommendation related to CAD data quality (PDQ). The "SASIG-PDQ" recommendation, published in 2002, defines a set of PDQ criteria used in the automotive industry. Thereafter, most of the CAD PDQ checker editors have implemented this set of criteria, and the recommendation was submitted to the ISO organization.

In terms of CAD data exchange format policy, 2 scenario need to be considered :

- OEM-Supplier relationship : in this case, as detailed previously, the CAD Data Exchange Policy applies. It specifies that the OEM native format (same release/version/service pack) must be used to minimize the impact of the data exchange on the data contents 
- OEM-OEM partnership : in this case, the CAD Data Exchange Policy doesn't apply. Neutral formats are used to exchange between different CAD systems, or different releases of the same CAD system (downward compatibility no ensured). Such a scenario implies a design master to consumer relationship.

\section{PDM Data Exchange}

PDM domain is less mature than the CAD domain in terms of data exchange. The exchange context is then much more complex than for the CAD data. Indeed, Each company implements their own PDM/PLM system customization, often complex, that is characterized by :

- Data model : add/modify attributes or objects,

- Rules and mechanisms applicable to attribute naming and codifications,

- Reference Breakdowns (functions, components) and classifications,

- Workflows and processes,

- Terminology/Vocabulary/Language,

- Integration with other systems : Manufacturing, Planning, ...

An illustration of PDM data exchange context is shown in Fig. 4 where both companies apply their own product data management rules.

\section{Company $A$}

\section{Reference : B024031972 (10 alphanumerical characters) \\ Version : 000 (3 numerical characters) \\ Decoupage : $\mathbf{G 1 1}$ (3 alphanumerical characters) \\ Designation : PEDALE-ACCELERATEUR (32 alphanumerical characters) \\ Explication : MODELE STANDARD (80 alphanumerical characters)}

Designation and Decoupage are dependent and driven by a classification system

Company $B$

\begin{tabular}{ll|}
\hline Identifier & : 47110-0H060 (11 alphanumerical characters) \\
Release & : 00A (3 alphanumerical characters) \\
Name & : BREAK-PEDAL ( 80 alphanumerical characters) \\
Description & : FRENCH MODEL (256 alphanumerical characters)
\end{tabular}

Fig. 4. Example of heterogeneous context for PDM data exchange

The usage of standards is the main way to exchange PDM data. Even if standards exist and are exhaustive, they cover a (too) wide perimeter, and their implementation remains complex. Nevertheless, data exchange solutions exist. They must be based on explicit use cases that define data exchange rules and processes, and the corresponding standard subset to be implemented. 
In terms of standards, STEP provides a set of application protocols that enable to exchange PDM data (structured data). For the engineering product development, STEP AP214 [6] is the relevant standard within the automotive industry. This standard covers a very wide scope, and is on way to be replaced by the standard STEP AP242, currently under development, nevertheless a fully compatible with STEP AP214. STEP AP214 covers a wide scope [1] including in particular product management data (item, version, approval, security, effectivity, date/organization/person), item definition structure (explicit assembly), item properties, work management (project activity, change management), classification, description of products with a large number of variants (diversity description, configuration), product breakdown (components, functions), reference mechanism to external documents, and process plan (process operations). Currently, only a very limited subset for STEP AP214 is widely implemented in commercial softwares. This scope covers mainly 3D geometric representation, PMI (Product Manufacturing Information or GD\&T) presentation, representation of explicit assemblies. It is estimated that it represents about $20 \%$ of the global scope of STEP AP214.

SASIG, consortium initially created to lead and support the development of the STEP AP214, has decided to develop a recommendation related to the exchange of PDM assemblies. The "SASIG-PDM Assembly Data Exchange" recommendation, published in 2003, defines how to exchange PDM assemblies with STEP AP214, with a proposal of process and STEP AP214 subset. Such a recommendation is destined to allowing assembly data exchange between PDM systems with an enriched content.

\section{STEP file content}

\begin{tabular}{|ll|}
\hline item.id & $:$ 47110-0X060 \\
item.name & $:$ BREAK-PEDAL \\
item.description & $:$ FRENCH MODEL \\
item_version.id & $:$ 00A \\
approval_status & $:$ approved \\
organization.name & $:$ CAR Corp \\
date_time & $:$ 2013.07.09 \\
\hline
\end{tabular}

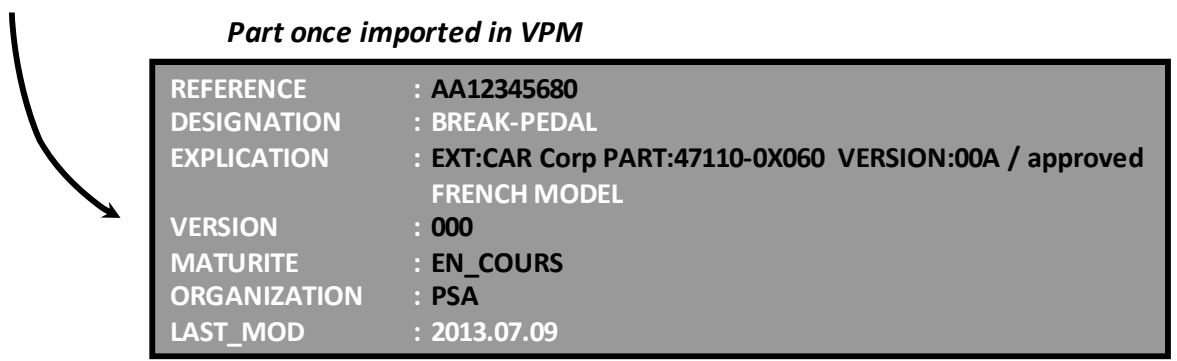

Fig. 5. Import of PDM data that are not compliant to my company codification rules

In terms of implementation, the capability to exchange with any partner is one of the success keys, whatever the used software. Indeed, a partner may have a PDM system or not, with a data exchange interface or not, but will always use a CAD 
system that enables to exchange 3D geometric representations and explicit assemblies in STEP format.

Thus, if we consider the export process, the experience shows that being able to export PDM data toward another PDM or CAD system enables to communicate with most of partners.

Then, if we consider the import process, generally the most complex, we need to consider 2 major cases that should enable to import any PDM data, whatever its contents :

- The partner uses the codification rules of my company. The import process should then be able to use all the attributes values provided in the exchanged package.

- The partner does not use the codification rules of my company. The import process should then create automatic values for the reference attributes (Unique identifier, Version), and the other attributes may be used as provided (see example in Fig. 5).

Once imported, depending on the business requirements, it is usually necessary to process those data in order to finalize their integration in the company IT environment (assign legacy reference, integration with other data, etc.).

\section{$5 \quad$ Long Term Archival and Retrieval}

For many years, the archival of engineering data has been reduced to the simple archival of drawings in the form of aperture cards or digital images. More globally, during the 2 last decades, the transition from the paper to the digital was observed within most of domains of management of product definition data. The automotive industry is now confronted with a situation, where product definition data covers a large diversity, and are managed by complex IT data structures, often with a strong adherence with solutions and their proprietary format [4]. In addition, we can observe that the required lifecycle of product definition data (more than 30 years) and the lifecycle of the used technologies (see Fig. 6) are not aligned.

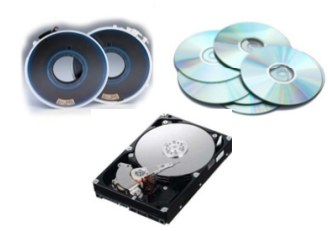

Storage media

$\sim 10$ years

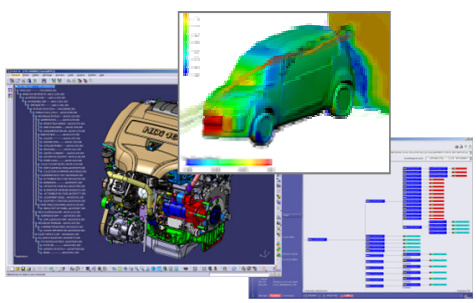

Software

3-7 years

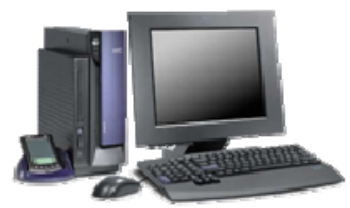

IT infrastructures

$<5$ years

Fig. 6. Lifecycle of technologies used in the management of product definition data 
The international automotive industry, following the aerospace industry [3] [8], has considered that those conditions cannot guarantee a consistent archival of its product definition data along its products lifecycle. In 2010, SASIG consortium decided to develop a set of recommendations that will guide companies to effective and efficient archival and retrieval practices. These recommendations, currently under development will include :

- A classification model of what data should be archived,

- A retention period recommendation,

- A quality assurance recommendation,

- A format recommendation,

- A LTAR process recommendation.

Data exchange standards are key elements to be considered in such recommendations. Thus, they provide a means to separate the data from their authoring softwares, and they have the biggest durability which is another important aspect in the LTAR domain [2].

At the moment, different major standards were identified by the SASIG-LTAR working group are :

- OAIS (ISO 14721 [7]) : The Open Archival Information System standard defines an open framework for the design and function of preservation repositories. This definition contains a reference model that defines the function, organization, and interaction of content within preservation repositories. OAIS should be considered as the basis of any archival system (see Fig. 7).

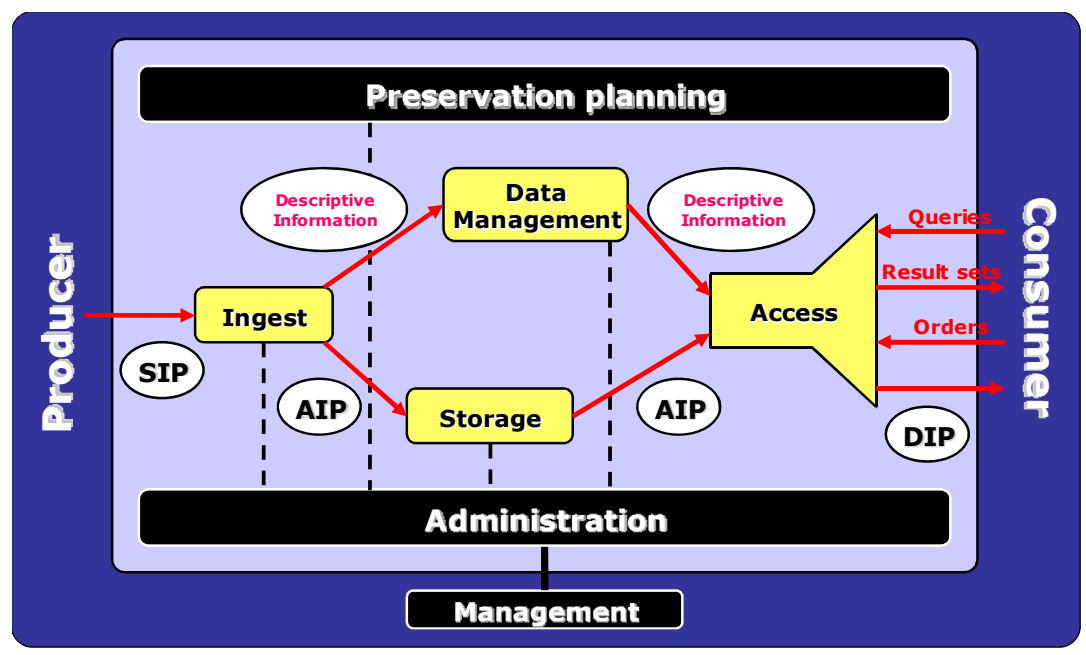

Fig. 7. OAIS Architecture 
- STEP Application Protocols (ISO 10303) : during the period between the archival and the retrieval, it is evident that a company will update or change its softwares. If we consider an archived data, it should be readable at any time along its lifecyle, independently of the software used at this moment. Therefore, it is the typical case of an exchange where the software used to create the data is not the same than the target one. The term of "Long Term Data Exchange" can then be used. In this context, data exchange standards are the most appropriate formats, taking into account the wide scope that they address.

- CAx-IF Recommended Practices and SASIG Recommendations that provide additional processes (CAD data quality) and mechanisms (Geometric and Assembly Validation Properties, etc.) very useful in that context.

The development activities of the recommendations are going on. The Format recommendation will be published by the end of 2013, and the complete set of recommendations by end of 2014. In between, a LTAR test pilot will be developed as proof of concept, promotion support.

\section{Conclusion}

Standards exist but due to their complexity, they are not controlled enough. The experience shows that guidelines, such as recommended practices and recommendations, are necessary for an appropriate usage of the standards. Thus, they provide clear use cases of the standard, completed with rules and proposal of process, aligned with the user requirements, and contribute to a better understanding and usage of the standards.

Nevertheless, even if the existing guidelines deployment is successful, guidelines and implementations are still missing in many domains such as configuration management (diversity dictionary, design use cases, etc.), product data management, requirements management, engineering change management, electrical harness design, etc. The corresponding deliverables should provide important inputs in the engineering data exchange and archival domains.

\section{References}

1. Chambolle, F.: Un modèle produit piloté par les processus d'élaboration : Application au secteur automobile dans l'environnement STEP. PhD Thesis, Ecole Centrale Paris (1999)

2. Kheddouci, F.: L'archivage à long terme de la maquette numérique 3D annotée. Master Degree, Ecole de Technologie Supérieure, Université de Québec, Canada (2010)

3. Kopena, J.B., Shaffer, J., Regli, W.C.: CAD Archives based on OAIS. In: ASME 2006 International Design Engineering Technical Conferences \& Computers and Information in Engineering Conference (2006)

4. Heutelbeck, D., Brunsmann, J., Wilkes, W., Hundsdörfer, A.: Motivations and Challenges for Digital Preservation in Design and Engineering. In: DP 2009, Austin, TX, USA (2009) 
5. Horwood, M., Kulkarni, S.: CAD data quality. The Journal of the Institution of Engineering Designers (2005)

6. ISO 10303-214, Industrial Automation Systems and Integration - Product Data Representation and Exchange, Part 214 : Application Protocol, Core Data for Automotive Mechanical Design Processes. ISO, International Organization for Standardization, Geneva (1999)

7. ISO 14721, Space data and information transfer systems - Open archival information system Reference model. ISO, International Organization for Standardization, Geneva (2003, 2012)

8. LOTAR Project, LOng Term Archiving and Retrieval, http: / / www . lotar-international .org

9. SASIG, Strategic Automotive product data Standards Industry Group, http: / /www.sasig.com

10. CAx Implementor Forum, http: //www. cax-if.org 